CrossMark < click for updates

Cite this: Chem. Commun., 2016, 52, 13417

DOI: $10.1039 /$ c6cc90497a

www.rsc.org/chemcomm

\section{Correction: Triplex-forming peptide nucleic acid modified with 2-aminopyridine as a new tool for detection of A-to-I editing}

\author{
Chiara Annoni, ${ }^{a}$ Tamaki Endoh, ${ }^{a}$ Dziyana Hnedzko, ${ }^{\mathrm{b}}$ Eriks Rozners ${ }^{\mathrm{b}}$ and \\ Naoki Sugimoto*ac
}

Correction for 'Triplex-forming peptide nucleic acid modified with 2-aminopyridine as a new tool for detection of A-to-I editing' by Chiara Annoni et al., Chem. Commun., 2016, 52, 7935-7938.

The authors regret that minor deviations in buffer $\mathrm{pH}$ due to a faulty $\mathrm{pH}$ meter have been identified in the experiments that provided the data presented in the original article, all measured $\mathrm{pH}$ values have since been found to be slightly higher than the true $\mathrm{pH}$ values. As the affinities of the triplex-forming peptide nucleic acids for both the wild-type and the edited RNA hairpins are sensitive to buffer $\mathrm{pH}$, the experiments furnishing the binding constants and other relevant data, reported in Fig. 1, Table 1 and Fig. 2 of the original article, as well as Fig. S2, S3, S6 and S7 and Tables S2-S5 of the original ESI, have been re-run. Revised versions of Fig. 1, Table 1 and Fig. 2 are included herein. The ESI attached to the original article has also been updated accordingly.

A)

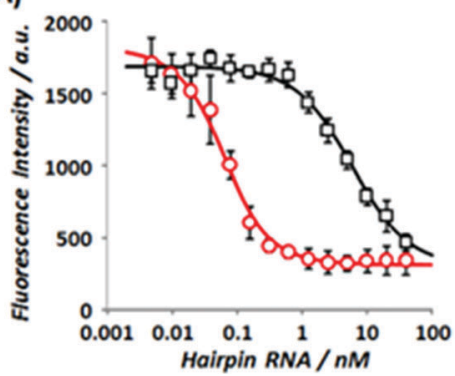

B)

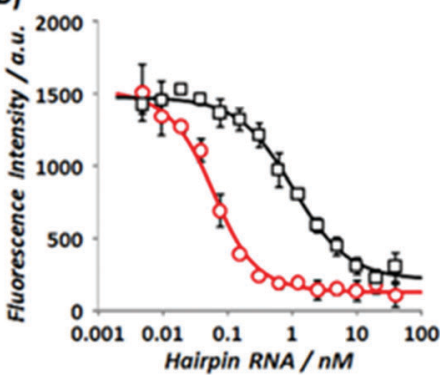

Fig. 1 Fluorescence of PNA-HF488 upon triplex formation at $25^{\circ} \mathrm{C}$ in a buffered solution at pH 7.0; (A) wild-type P5AC (black squares) and edited P5IC (red circles) hairpin RNAs, and (B) wild-type P5AU (black squares) and edited P5IU (red circles) hairpin RNAs.

Table $1 K_{\text {A25 }}$ values for the interaction of PNA-HF488 with hairpin RNAs at indicated $\mathrm{pH}$ values obtained by fluorescence titration at $25{ }^{\circ} \mathrm{C}^{a}$

\begin{tabular}{|c|c|c|c|c|c|c|}
\hline Hairpin RNA & \multicolumn{6}{|c|}{$\underline{K_{\mathrm{A} 25}\left(\times 10^{9} \mathrm{M}^{-1}\right)}$} \\
\hline P5AC & $8.66 \pm 1.8$ & $2.44 \pm 0.44$ & $0.522 \pm 0.02$ & $0.173 \pm 0.02$ & $0.051 \pm 0.02$ & n.d. ${ }^{b}$ \\
\hline P5AU & $63.8 \pm 11$ & $27.0 \pm 2.4$ & $7.82 \pm 0.62$ & $0.971 \pm 0.05$ & $0.157 \pm 0.04$ & n.d..$^{b}$ \\
\hline P5IU & $101 \pm 17$ & $106 \pm 16$ & $82.1 \pm 12$ & $29.5 \pm 1.4$ & $5.28 \pm 0.56$ & $1.18 \pm 0.12$ \\
\hline
\end{tabular}

${ }^{a}$ The reported values are means \pm standard deviations from at least three independent measurements. ${ }^{b}$ The value could not be determined due to low affinity.

\footnotetext{
${ }^{a}$ Frontier Institute for Biomolecular Engineering Research (FIBER), Konan University, 7-1-20 Minatojima-minamimachi, Chuo-ku, Kobe 650-0047, Japan.

E-mail: sugimoto@konan-u.ac.jp

${ }^{b}$ Department of Chemistry, Binghamton University, State University of New York, Binghamton, New York 13902, USA

${ }^{c}$ Faculty of Frontiers of Innovative Research in Science and Technology (FIRST), Konan University, 7-1-20 Minatojima-minamimachi, Chuo-ku, Kobe 650-0047, Japan
} 
A)

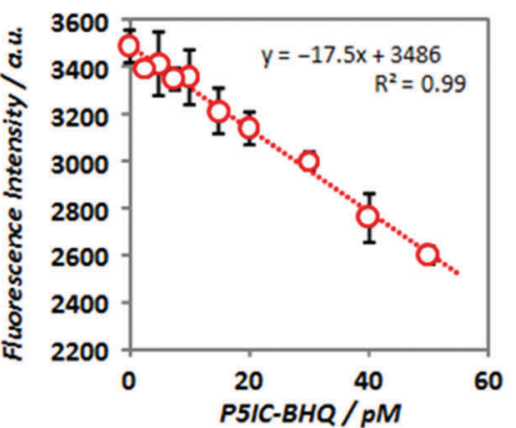

C)

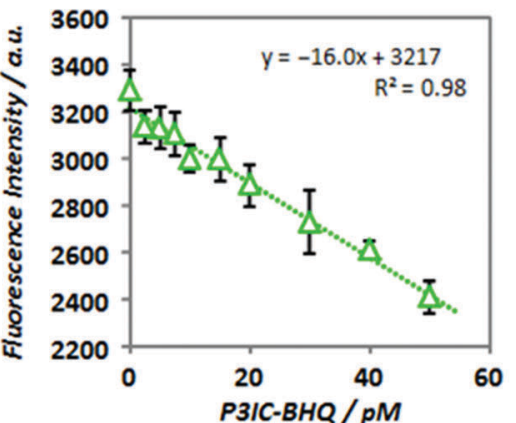

B)

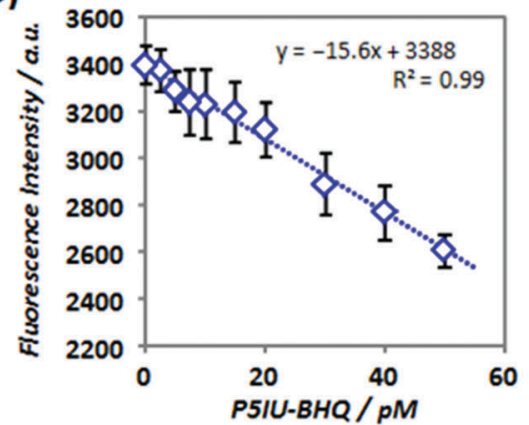

D)

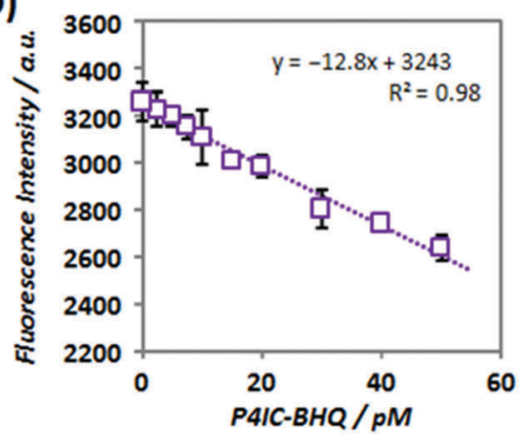

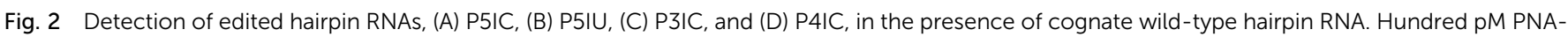

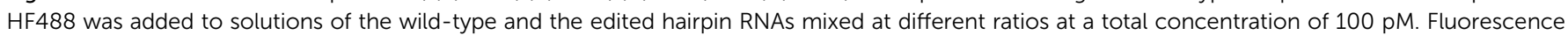

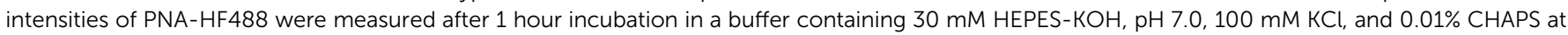

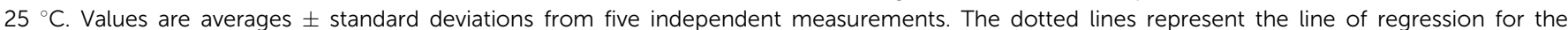
calibration curve. The regression equation and the $R$-squared values are displayed in the charts.

As a result of the identified errors, the originally quoted limit of detection (LOD) values for the inosine-containing hairpin RNAs, P3IC, P4IC, P5IC and P5IU, are slightly increased to 17.7, 23.7, 13.0, and 17.3 pM, respectively (Table S4), but still remain 1000 times lower than the reported experimental conditions adopted by fluorometric methodology.

The Royal Society of Chemistry apologises for these errors and any consequent inconvenience to authors and readers. 